\title{
Lev Landau and the conception of neutron stars
}

\author{
Dmitry G. Yakovlev ${ }^{1}$, Paweł Haensel ${ }^{2}$, Gordon Baym ${ }^{3}$, Christopher J. Pethick ${ }^{4,5}$ \\ ${ }^{1}$ Ioffe Physical Technical Institute, Politekhnicheskaya 26, 194021 St.-Petersburg, Russia \\ ${ }^{2}$ N. Copernicus Astronomical Center, Bartycka 18, 00-716 Warsaw, Poland \\ ${ }^{3}$ Department of Physics, University of Illinois, 1110 W. Green Street, Urbana, Illinois 61801, USA \\ ${ }^{4}$ Niels Bohr International Academy, Niels Bohr Institute, Blegdamsvej 17, DK-2100, Copenhagen, Denmark \\ ${ }^{5}$ NORDITA, Royal Institute of Technology and Stockholm University, \\ Roslagstullsbacken 23, SE-10691 Stockholm, Sweden
}

\begin{abstract}
We review the history of neutron star physics in the 1930s that is related to L. Landau. According to recollections of Rosenfeld (1974, Proc. 16th Solvay Conference on Physics, p. 174), Landau improvised the concept of neutron stars in a discussion with Bohr and Rosenfeld just after the news of the discovery of the neutron reached Copenhagen in February 1932. We present arguments that the discussion took place in March 1931, before the discovery of the neutron, and that they in fact discussed the paper written by Landau in Zurich in February 1931 but not published until February 1932 (Phys. Z. Sowjetunion 1 285). In his paper Landau mentioned the possible existence of dense stars which look like one giant nucleus; this can be regarded as an early theoretical prediction or anticipation of neutron stars, prior to the discovery of the neutron. The coincidence of the dates of the neutron's discovery and the paper's publication has led to an erroneous association of the paper with the discovery of the neutron. In passing, we outline the contribution of Landau to the theory of white dwarfs and to the hypothesis of stars with neutron cores.
\end{abstract}

\section{Introduction}

Neutron stars, highly compact objects with a mass of order that of the Sun but only about $10 \mathrm{~km}$ in radius, are the engines of many remarkable astrophysical phenomena, from radio pulsars to compact $\mathrm{X}$-ray sources. These objects are not only fascinating in themselves, but they have been the basis of critical tools in astrophysical measurements; in addition they provide a window into the properties of matter at the highest densities. Although neutron stars were first discovered as radio pulsars in 1967 [1, they had been the subject of theoretical investigations since the 1930s, and had been studied in the hope of resolving fundamental questions of the final states of stellar evolution, the source of the energy released in supernovae, and even the energy source of ordinary stars. In these initial developments the great Soviet physicist, Lev D. Landau (1908-1968), played a controversial role. Our focus in this paper is to clarify Landau's contributions to neutron stars while summarizing the early history of the physics that fed into the idea of neutron stars. A brief summary of this investigation was given in Ref. [2].

To set the stage, we recall an oft-quoted reminiscence of Léon Rosenfeld at the 1973 Solvay Conference. He described a discussion between Niels Bohr, Landau, and himself in Copenhagen in early 1932 [3]:

I recall when the news on the neutron's discovery reached Copenhagen, we had a lively discussion on the same evening about the prospects opened by this discovery. In the course of it Landau improvised the conception of neutron stars - 'unheimliche Sterne', weird stars, which would be invisible and unknown to us unless by colliding with visible stars they would originate explosions, which might be supernovae. Somewhat later, he published a paper with Ivanenko in which he again mentioned neutron stars as systems 'to which quantum mechanics would not be applicable.'

This story was first repeated by Baym and Pethick [4, and later by Baym [5], as well as by Shapiro and Teukolsky in their monograph [6] (first footnote on p. 242). It naturally created the impression, widely 
mentioned in the literature, that neutron stars were predicted by Landau in 1932 as a direct consequence of the discovery of the neutron. The historical facts do not bear the story out.

James Chadwick, who discovered the neutron in February 1932, reported his finding to Bohr in a letter dated 24 February 1932 (Fig. 1) which would date this purported evening discussion to shortly afterwards. However, as is often the case in recollections by participants many years after the fact, Rosenfeld's account is only a fuzzy hint of the actual story. On the one hand, Landau was not in Copenhagen in 1932. Furthermore, neither were Bohr nor Rosenfeld in Copenhagen when Chadwick's letter arrived; both were travelling, and Chadwick's letter apparently lay on Bohr's desk until Bohr returned in the second half of March. As we discuss below in Sec. 3, Landau had already had the idea of the possibility of matter in a star "forming one gigantic nucleus," a year earlier. In addition he never wrote a paper with the Soviet physicist Dmitry Ivanenko on stars (e.g., Ref. [7]); most likely Rosenfeld was thinking of Landau's earlier paper (Sec. 6). Nonetheless, Rosenfeld's recollection does provide a useful stepping stone into the early history of neutron stars.
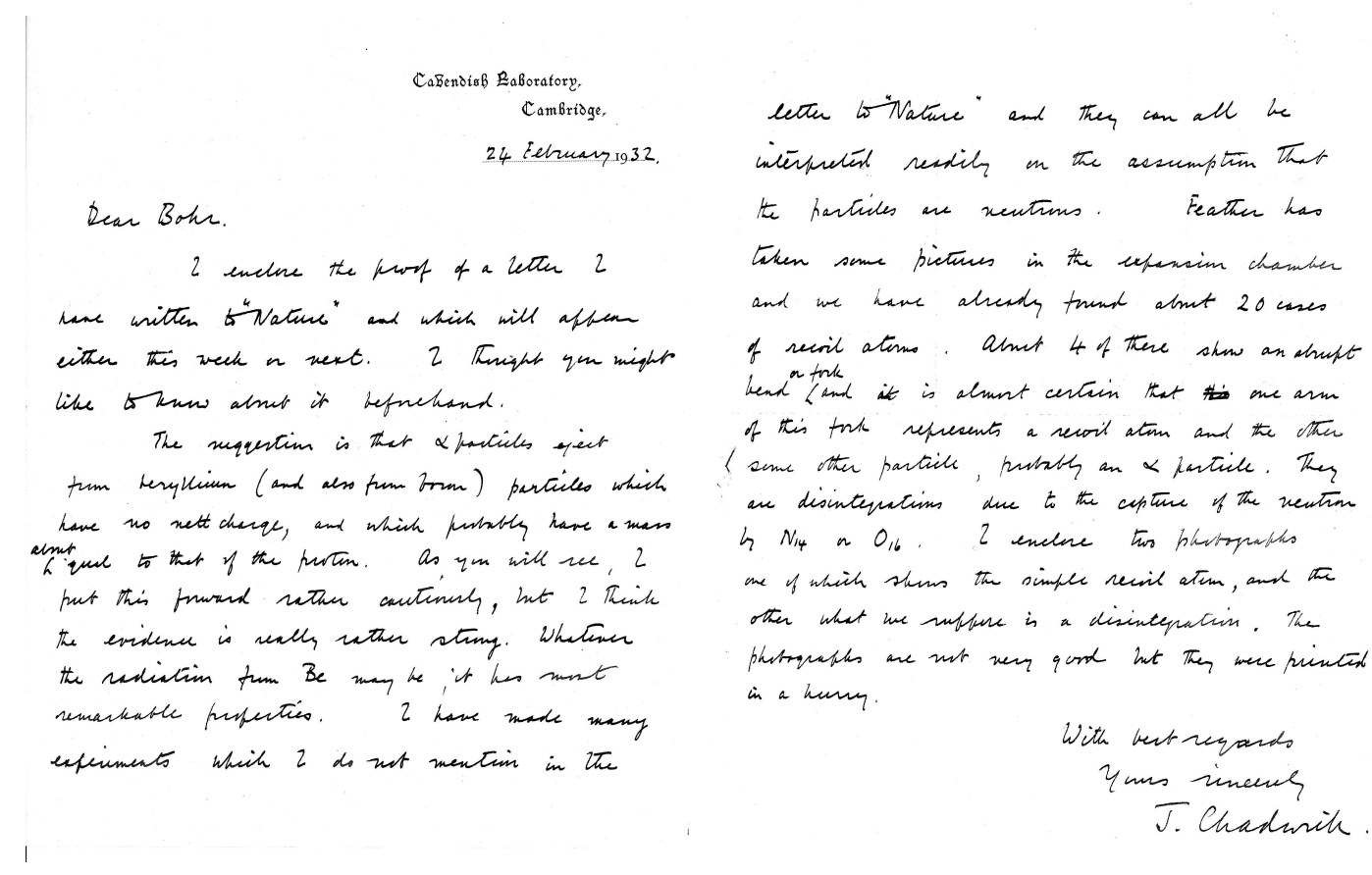

Figure 1: Chadwick's letter to Bohr on the discovery of the neutron. Courtesy of the Niels Bohr Archive, Copenhagen. Reproduced with the kind permission of Judith Chadwick.

\section{Landau and Bohr}

Landau's trajectory through Europe and his interactions with Bohr are crucial to understanding the correct history of Landau's connection to neutron stars. Landau graduated from Leningrad State University in January 1927, at the age of 19, and was appointed to a position, resembling that of a present day graduate student, at Leningrad Physical Technical Institute (now the Ioffe Physical Technical Institute, St. Petersburg). His supervisor was Yakov (or Jakob) I. Frenkel, head of the Theoretical Physics De- 
partment. A brilliant student, Landau was awarded one of two stipends of the Ministry of Education (the 'Narodnyi Komissariat Prosvyastcheniya', or the 'People's Commissariat of Enlightenment') for a one and a half year trip abroad for scientific work.

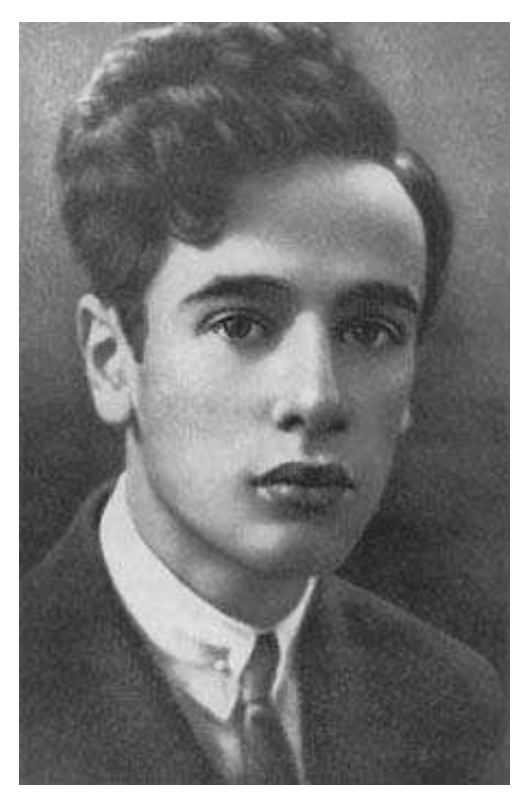

Figure 2: Lev Landau in 1929.

Landau, seen in Fig. 2] at the time (from Ref. 8), started his trip in October 1929, visiting numerous physics centers in Europe including (chronologically) Berlin, Göttingen, Leipzig, Zurich, Copenhagen, Cambridge, and Copenhagen, Zurich, and Copenhagen again. In particular, he visited Bohr in Copenhagen 8 April - 3 May 1930, 20 September - 20 November 1930, and 25 February - 19 March 1931 (Ref. 9, footnote on p. 359). Landau liked Bohr and looked upon him as his only teacher; Bohr returned the friendship. In the beginning of 1931, when Landau's Soviet stipend had run out, Bohr helped him obtain a Rockefeller Fellowship which allowed Landau to prolong his stay and visit Bohr in Copenhagen the third time. Figure 3 is a photo of Niels Bohr's annual conference in Copenhagen in 1930 with Landau in the front row on the right. After the third visit Landau returned to Leningrad and moved to Kharkov the same year.

Landau and Bohr met again in Copenhagen and the USSR. Landau visited Bohr in Copenhagen 18 September - 3 October 1933 and 1 June -8 July 1934, to attend conferences organized by Bohr. After 1934, Landau did not travel abroad (except for medical treatment in Czechoslovakia in 1963 after his terrible car accident on 7 January 1962, which terminated his scientific career). Bohr visited the USSR and met with Landau there three times: in May 1934 (Leningrad, Moscow, Kharkov), June 1937 (in Moscow, during Bohr's half-year tour to Japan, the USA, China, and the USSR), and May 1961 (Moscow) - see Ref. [9, pp. 415, 417, and 528. 


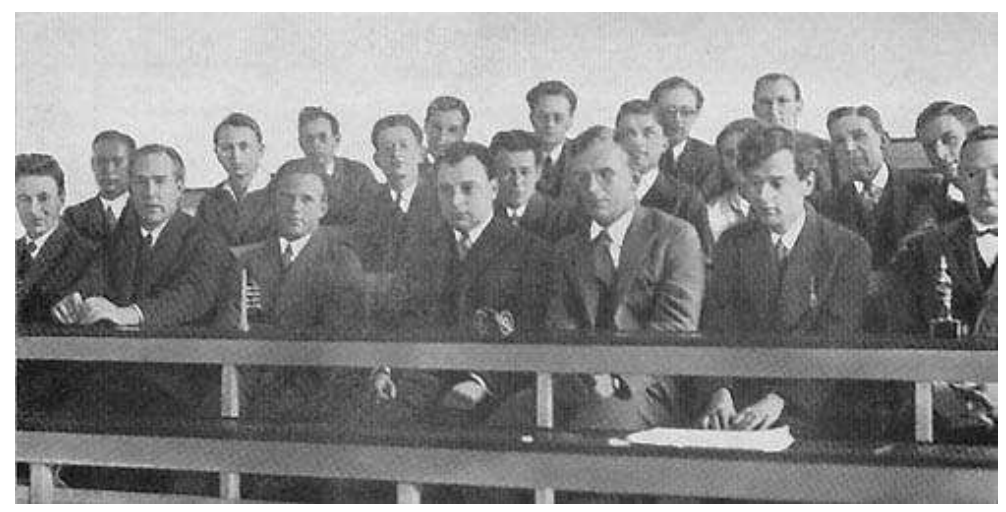

Figure 3: The annual conference at the Institute for Theoretical Physics in Copenhagen in 1930. First row (from left to right): O. Klein, N. Bohr, W. Heisenberg, W. Pauli, G. Gamow, L. Landau, H. Kramers. Courtesy of the Niels Bohr Archive, Copenhagen.

\section{Landau's paper 'On the theory of stars'}

On 7 January 1932, more than one month before Chadwick's announcement of the discovery of the neutron, and even before Chadwick started his successful experiment (see Sec. 44), Landau submitted a paper [10] in English to the Physikalische Zeitschrift der Sowjetunion, the first Soviet physical journal published in languages other than Russian. The paper was published on 29 February 1932, less than a week after Chadwick's letter to Bohr. Interestingly, the last line of the published paper says: 'February 1931, Zurich,' from which one would conclude that Landau wrote the paper one year before the discovery of the neutron, in Zurich, but for unknown reasons had delayed sending it to a journal for nearly a year 1 The omission of the February 1931 date in reprints of Landau's paper [12, 13, aided by Rosenfeld's recollection, has led to an erroneous association, of Chadwick's discovery with Landau's early ideas on dense stars (see Sec. 6).

The four page paper, written in Landau's concise style, consists of two parts, both of which are significant. The first part is devoted to an incisive calculation of the maximum mass of a white dwarf, and the second to speculations on the structure and physics of stars of higher density. The maximum mass is the so-called Chandrasekhar mass limit of white dwarfs, the highest mass supportable against gravity by the pressure of relativistic degenerate electrons. The history of the theoretical prediction of this mass is described, for instance, by Nauenberg [14] and Shaviv [15]. The problem was a forefront application of quantum statistical mechanics to the open question of the nature of white dwarfs, but it is not clear to what extent Landau was aware of the developments that were taking place 2 Landau calculated the maximum mass independently but later than Chandrasekhar. He presents an important simple formula,

$$
M_{0}=\frac{3.1}{m^{2}}\left(\frac{\hbar c}{G}\right)^{3 / 2},
$$

relating the maximum mass to four physical parameters - Planck's constant $\hbar$, the speed of light $c$,

\footnotetext{
${ }^{1}$ Karl Hufbauer [11] speculates that, "He probably intended to publish it in Nature, the field's leading popular venue. As things turned out, however, he ended up carrying the manuscript back to the Leningrad Physico-Technical Institute when he returned to the Soviet Union that spring and submitted it to the new Physikalische Zeitschrift der Sowjetunion early the next year."

${ }^{2}$ The first estimates of a maximum mass were made by Wilhelm Anderson [16] and then by Edmund Clifton Stoner [17. The exact calculation of the maximum mass was first carried out by Chandrasekhar in July 1930, without knowledge of Anderson's and Stoner's works, and was published in Ref. [18].
} 
Newton's gravitational constant $G$, and $m$, the mass of the matter per electron. For white dwarf interiors one has $m \approx 2 m_{p}$, with $m_{p}$ the proton mass, which gives the well known value $M_{0} \approx 1.44 M_{\odot}$, where $M_{\odot}$ is the mass of the Sun. Note that Landau gives $M_{0} \approx 1.5 M_{\odot}$ (while Chandrasekhar [18 originally obtained $M_{0} \approx 0.91 M_{\odot}$ because he assumed an unreasonably high value of $m=2.5 m_{p}$, as explained, e.g., in Ref. [15]). Landau did not state it explicitly, but his formula shows that the scale of the mass of white dwarfs is given by the proton mass, $m_{p}$, times $\alpha_{G}^{-3 / 2} \approx 10^{57}$, where $\alpha_{G}=m_{p}^{2} G / \hbar c$ is the modern 'gravitational fine structure constant'.

Landau next discusses more massive stars. Although he would not write on neutrons in stars until nearly six years later, he was confronting at this time the theoretical question of the collapse of stars of mass greater than $M_{0}$ to higher and higher densities, and was toying with ideas of heavier stars having cores in which even "the laws of ordinary quantum mechanics break down...".

Landau's colleague and friend, Matvei P. Bronstein, a remarkably talented theoretical physicist with very broad interests from solid-state physics to quantum gravity (and sadly shot in 1938 by the Stalin regime), expanded [19] on how Landau was thinking. While the problem of the source of energy in stars was unsolved at the time, the great difference between ordinary main sequence stars and white dwarfs was not clear. To Landau the energy problem appeared to be an obstacle that demanded new physical theories going beyond standard quantum mechanics. Landau (wrongly) assumed that the maximum mass limit, $1.5 M_{\odot}$, he calculated was the maximum for all stars to which standard physics was applicable. The possibility that massive ordinary stars were composed of low-density non-degenerate matter was not taken into account. Furthermore, observational evidence on the existence of many massive stars was limited. Any star with $M$ greater than $1.5 M_{\odot}$ was treated as 'pathological,' containing a core of very dense matter, where standard physical laws (including energy conservation) were violated; he also assumed that dense 'pathological' regions could appear in less massive stars. Landau writes,

As we have no reason to believe that stars can be divided into two physically different classes according to the condition $M>$ or $<M_{0}$, we may with great probability suppose that all stars possess such pathological regions. It does not contradict the above arguments, which prove only that the condition $M>M_{0}$ is sufficient (but not necessary) for the existence of such regions. It is very natural to think that just the presence of these regions makes stars stars. But if it is so, we have no need to suppose that the radiation of stars is due to some mysterious process of mutual annihilation of protons and electrons, which was never observed and has no special reason to occur in stars. Indeed we have always protons and electrons in atomic nuclei very close together, and they do not annihilate themselves; and it would be very strange if the high temperature did help, only because it does something in chemistry (chain reactions!). Following a beautiful idea of Professor Niels Bohr's we are able to believe that the stellar radiation is due simply to a violation of the law of energy, which law, as Bohr has first pointed out, is no longer valid in the relativistic quantum theory, when the laws of ordinary quantum mechanics break down (as it is experimentally proved by continuous-rays spectra and also made probable by theoretical considerations [20]). We expect that this must occur when the density of matter becomes so great that atomic nuclei come in close contact, forming one gigantic nucleus.

Landau's understanding that protons and electrons constitute atomic nuclei and do not annihilate there provides additional proof that the paper was conceived before the discovery of the neutron. While the suggestion that sources of stellar energy were located in 'pathological cores' was convenient, it was naive. Furthermore, that the energy output of stars could be attributed to a breakdown of energy conservation in quantum mechanics, while a plausible conjecture at the time, is, as we now know, wrong. But Landau's anticipation of dense (neutron) stars which look like giant atomic nuclei was prescient. 


\section{The discovery of the neutron}

The neutron, which was predicted as early as 1920 by Rutherford [21, was finally discovered by Chadwick 22], who left a colorful description of his discovery 23. After Rutherford's prediction Chadwick had attempted to find evidence of the neutron many times but invariably failed. At the beginning of 1932 he became inspired by the paper of Curie and Joliot 24, published on 28 January. As acknowledged in Chadwick's second publication on the neutron's discovery [25], experiments similar to that of Curie and Joliot were conducted also by Bothe and Becker [26], Curie [27] and Webster [28, all of whom reported results of bombardment of beryllium nuclei by alpha particles produced by a polonium source. They found that neutral reaction products (which they interpreted as gamma rays) knocked out protons from paraffin. Chadwick and Rutherford did not believe this interpretation. Chadwick immediately went to work, conducting a similar experiment with better equipment and in a few days of strenuous work found the same result 23. However, he interpreted it correctly, as production of neutrons (not gamma-rays) in $\alpha+$ Be collisions. Finally successful, he could even estimate, using energy and momentum conservation in the knock-out reaction, the mass of the neutron, which he found to be close to the proton mass. His discovery [22, announced in Nature, was received by the editor on 17 February and published on 27 February 1932. More detailed explanations were given in the next paper [25].

Note that the first theoretical model of atomic nuclei as composed of protons and neutrons was suggested in April 1932 by Ivanenko [29].

\section{The prediction of neutron stars after the neutron's discovery}

The first explicit prediction of neutron stars was made by Walter Baade and Fritz Zwicky at Caltech in December 1933, nearly two years after the discovery of the neutron, in trying to explain the enormous energy released in supernova explosions. Reporting their results at the meeting of the American Physical Society at Stanford on 15-16 December 1933, they wrote with remarkable foresight [30, "with all reserve we advance the view that supernovae represent the transition from ordinary stars into neutron stars, which in their final stages consist of closely packed neutrons." They amplified this indeed accurate picture in the second and third of their papers [31, 32] of the following March which proposed cosmic-ray production in supernovae, "Such a star may possess a very small radius and an extremely high density. As neutrons can be packed much more closely than ordinary nuclei and electrons, the 'gravitational packing' energy in a cold neutron star may become very large, and, under certain circumstances far exceed the ordinary nuclear packing fractions. A neutron star would therefore represent the most stable configuration of matter as such." Only through such close packing could they understand the energy release in supernovae which they estimated to be equivalent to the annihilation of the order of several tenths of a solar mass. However, they could only guess at the scenario for forming neutron stars. In their fourth publication Baade and Zwicky [33] naively (and wrongly) explain that, "... neutrons are produced on the surface of an ordinary star" [under the effect of cosmic rays] and "rain' down towards the center as we assume that the light pressure on neutrons is practically zero."

Their idea of neutron stars quickly made it into the popular press, as Fig. 田 from The Los Angeles Times illustrates 3 The stellar diameter mentioned in the cartoon is uncannily accurate.

\section{Revisiting Rosenfeld's reminiscence}

With this history in place we ask how we can make sense of Rosenfeld's recollection. First, as we have seen, there was no connection between the discovery of the neutron and Landau's concept of dense stars.

\footnotetext{
${ }^{3}$ We thank Janusz Ziółkowski for calling this cartoon, presented in a talk of Robert P. Kirshner at the Eight Texas Symposium on Relativistic Astrophysics [34, to our attention. We are also grateful to R. P. Kirshner for helpful information referring to this cartoon.
} 


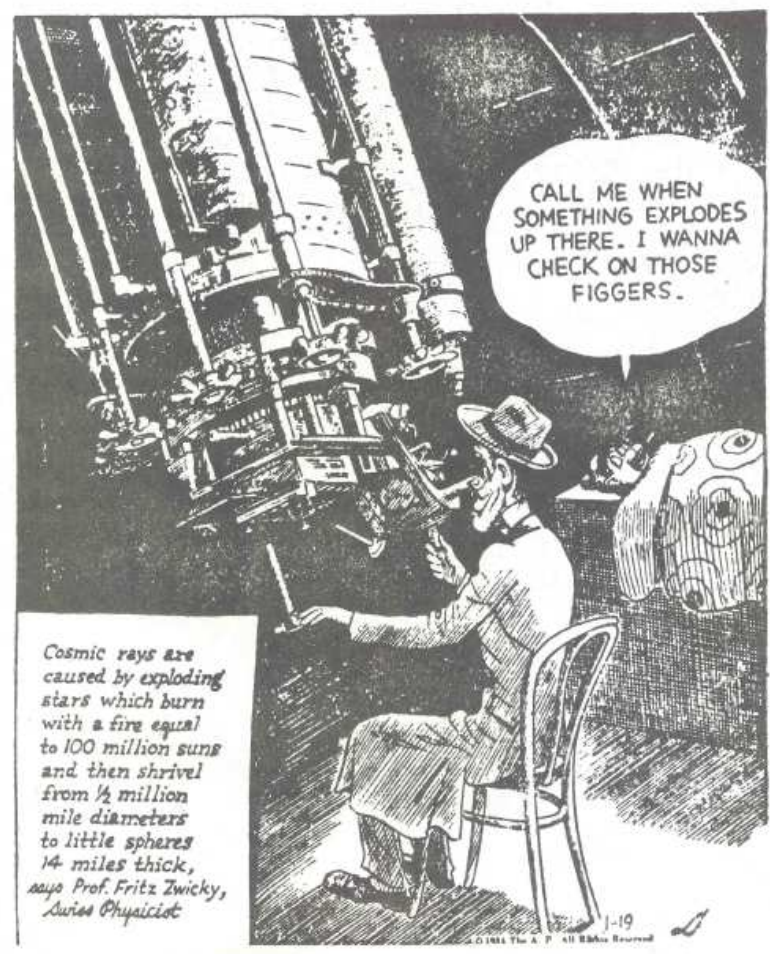

Figure 4: Baade and Zwicky's prediction of neutron stars, reported in this cartoon in the Los Angeles Times of 19 January 1934. The lower box reads: "Cosmic rays are caused by exploding stars which burn with a fire equal to 100 million suns and then shrivel from 1/2 million mile diameters to little spheres 14 miles thick, says Prof. Fritz Zwicky, Swiss Physicist." Reproduced with permission of The Associated Press, Copyright (C)1934. All rights reserved.

Thus Landau clearly did not improvise the concept of unheimliche Sterne in a discussion with Bohr and Rosenfeld. Most likely the discussion between Landau, Bohr, and Rosenfeld took place during Landau's third trip to Copenhagen in February and March 1931, just after he wrote his paper in Zurich. It was natural that Landau at this point would discuss his paper with Bohr and Rosenfeld (the latter had arrived in Copenhagen on 28 February 1931, see Ref. [35]), since Landau had tried to utilize Bohr's ideas of violation of traditional physics. Then in using the term unheimliche Sterne Rosenfeld was referring to Landau's idea of a star as a single dense nucleus in which quantum mechanics is violated. Once the neutron was discovered there was no longer a need to violate quantum mechanics to avoid unobserved electron-proton annihilations. Given the compelling arguments that the discussion between the three took place just after Landau's arrival in Copenhagen in 1931, it is unlikely that it happened at a later occasion when all three were together - e.g., 18 September - 3 October 1933 or 1 June - 8 July 1934 in Copenhagen, or during one of the two trips by Bohr to the Soviet Union in 1934 and 1937. In 1931 unheimliche Sterne would have referred simply to a star as a single dense nucleus, more like neutron stars as we know them.

There is no evidence that Landau would have discussed unheimliche Sterne as being "invisible and unknown to us unless by colliding with visible stars they would originate explosions, which might be supernovae." And Rosenfeld's reference to Landau publishing a paper with Ivanenko must have been to Landau's 1931 paper. The name of Ivanenko could well have been mentioned in association with Ivanenko's paper [29] on the proton-neutron structure of atomic nuclei written after the neutron's discovery. 


\section{Stars with neutron cores}

The source of stellar energy remained unknown until 1938 (see, e.g., Ref. [15]). In addition the ultimate fate of stars more massive than $M_{0}$ remained a theoretical mystery. One suggestion was that they developed cores of highly compressed matter (see e.g., Ref. 36). While Chandrasekhar in late 1934 at Trinity College had given serious arguments against this possibility, suggesting mass loss or supernova phenomena instead [37, 38, Gamow [39, reviewing Chandrasekhar's (1931) and Landau's (1932) earlier arguments, wrote that more massive stars "are subject to the formation of matter in the nuclear state in their interior at some period of their existence." This state would be formed by beta capture of electrons by nuclei, changing protons to neutrons. [Although Gamow did not refer to it, the first microscopic descriptions of the equation of state of nuclear matter in beta equilibrium had actually been given by Hund [40] a year earlier.] Such a state would not only provide, through gravitational release, energy "enough to secure the life of the star for a very long period of time," but eruptive processes at the surface of the dense nuclear core could give rise to spewing out of "nuclear substance" and formation of various nuclei, thus helping with problems of nucleosynthesis.

Quite independently, Landau, addressed the origin of stellar energy in a paper first published in Russian in the Proceedings of USSR Academy of Sciences [41] in 1937, and then in Nature [42] (submitted from Moscow via Bohr in Copenhagen in late 1937). Landau proposed that in ordinary stars of masses greater than a critical mass $\sim 0.001 M_{\odot}$, a neutron core, "where all the nuclei and electrons have combined to form neutrons," would be energetically favored over a normal core. For Landau, it was a step from "pathological" cores in his paper written in 1931 with unknown physics to neutron cores described by standard physics. The stellar envelope contracts (accretes) slowly onto the compact core. The huge release of gravitational energy (due to the core compactness) in the course of accretion would be sufficient to heat the star and support its luminosity at the high level for a long time. In particular, Landau finished his new paper with an appeal to build up the models of such stellar objects. Hund's earlier work is referred to here, but only as an afterthought at the suggestion of Bohr and Møller 43. Landau stressed, as did Gamow, that a neutron core would "give an immediate answer to the question of the sources of stellar energy."

We note in passing that the political situation in the Soviet Union had turned from bad to worse in 1937, and Landau was in real danger of arrest. To avoid this, P. L. Kapitsa organized a campaign to popularize Landau's work and make Landau a famous scientist (including very favorable press coverage; see Hufbauer [11] for an amusing story about the publication of the new paper). However the political regime disregarded such campaigns. Landau was arrested in 1938 in Moscow for 'anti-Soviet activity' and spent one year in prison. He was released (and the conditions of his sentence were softened) mainly due to the efforts of Kapitsa who did not fear to write several letters in Landau's defense to Stalin and top Soviet officials 44. Curiously, in his first letter to Stalin on 28 April 1938, the very day of the Landau's arrest, Kapitsa argued that Landau "... published one remarkable paper, where he was the first to show a new source of stellar radiation." Thus Landau's paper was used as an argument to save his life.

When Landau was released, it was already evident (e.g., Ref. [45) that normal stars support their life by nuclear burning. The problem of stellar energy was solved and Landau's appeal was temporarily forgotten. However the idea was realized later in the form of Thorne-Żytkow objects, hypothetical stellar objects that from the outside look like ordinary giants or supergiants. Inside, they contain large rarefied convective envelopes and dense degenerate cores, again like giants and supergiants. However, ordinary giants and supergiants possess degenerate electron cores (i.e., contain white dwarfs in their centers) while these new objects possess much denser, neutron degenerate cores (i.e., neutron stars). The idea of constructing models of red giants or supergiants, replacing the electron degenerate cores by compact neutron cores, was discussed by several authors, including Fermi, Paczyński, Ostriker, Bisnovatyi-Kogan, Sunyaev, and Thorne. For references, see the paper by Thorne and Żytkow 46. Useful references are also given in [47. Thorne and Żytkow wrote a very detailed paper [46] where they built the first models of such objects. The theory of Thorne-Żytkow objects was also studied in Refs. [47, 48, 49]. 
Unfortunately, accurate modeling of Thorne- $\dot{Z}$ ytkow objects is extremely difficult. The main problem is to describe the energy release, loss and transfer throughout the object, from its center, to the outer layers of the neutron star, and through the rarefied convective envelope. The most difficult place is near the bottom of the convective envelope (close to the outer layers of the 'inner' neutron star). This layer is hot, neutrino emission carries away a lot of energy, and convection is highly unusual. A small increase in the neutrino losses may reduce the flow of heat from the neutron star to the convective envelope, quench the convection, and the object will be destroyed. Variations of thermal conductivity and nuclear reaction rates under highly unusual conditions in these objects can also be harmful. We add that there is no solid observational evidence that these objects exist. Therefore, the existence of Thorne-Żytkow objects remains an open question.

\section{Summary}

We have outlined the early history of the theory of neutron stars and Landau's contribution to that theory (see also the chronology of events in the Appendix). Our key point is that in Zurich, in February 1931, one year before the discovery of the neutron, Landau wrote a remarkable paper [10. In that paper he calculated the maximum mass of white dwarf stars (independently of, but later than, Chandrasekhar) and predicted the existence of dense stars which look like giant atomic nuclei (a valid description of neutron stars nowadays). He suggested also that the laws of quantum mechanics are violated in these very dense stars. Let us be merciful with Landau for that suggestion - he was only 23 years old! Landau discussed his paper with Bohr and Rosenfeld in Copenhagen in the period from 28 February to 19 March 1931, and published it in February 1932, just when the discovery of the neutron was announced. An accidental coincidence of dates of the neutron's discovery and the publication of Landau's paper produced confusion in the literature, with attempts to claim that Landau predicted neutron stars just after learning about the existence of the neutron. This claim seems definitely invalid.

The question of whether Landau predicted neutron stars cannot be answered unambiguously. On the one hand, yes, he predicted them, but without neutrons and by violating quantum mechanics. On the other hand, no, his vision of dense stars was too far from our knowledge today. Perhaps, it would be more appropriate to say that he superficially anticipated them. However, we do not insist and allow the reader to judge. The next prediction of neutron stars by Baade and Zwicky 30] was certainly more realistic.

In 1937 Landau also contributed to the idea of stars with neutron cores, which corresponded to what would later be called Thorne-Żytkow objects. They are very difficult to model and have not been observed.

After the car accident in 1962, Landau did not fully recover and, being in constant pain, he could not concentrate on science. He died on 1 April 1968, just as the discovery of neutron stars as radio pulsars, announced in Nature [1] on 24 February 1968, was being widely discussed. Even if he was told about that discovery, he was certainly not interested in it and could not understand the importance of his contribution to the physics of compact stars. It was our aim to remind the reader of this contribution and extract its essence.

Acknowledgement. We greatly thank Finn Aaserud and Felicity Pors of the Niels Bohr Archive in Copenhagen for tracing parts of this history. We are also grateful to Gennady Gorelik and Alexei Kojevnikov for useful comments, and to Nicolas Chamel, Yurii N. Gnedin and Peter Shternin for their help in finding old publications. 


\section{Appendix. Chronology}

1. February 1931, Zurich. Landau finishes his paper [10, in which he calculates the maximum mass of white dwarfs and predicts the existence of dense stars which look like giant atomic nuclei.

2. 25 February - 19 March, 1931. Landau in Copenhagen. He most likely discusses his paper with Bohr and Rosenfeld in the period from 28 February (when Rosenfeld arrives) to 19 March.

3. 7 January 1932. Landau submits his paper [10 to Physikalische Zeitschrift der Sowjetunion.

4. End of January 1932. Chadwick became interested in conducting the experiment which led to the discovery of the neutron.

5. 17 February 1932. Chadwick submits his paper 22] on the discovery of the neutron to Nature.

6. 24 February 1932. Chadwick writes a letter to Bohr informing him of the discovery of the neutron.

7. 27 February 1932. Chadwick's paper on the discovery of the neutron is published.

8. 29 February 1932. Landau's paper [10 published.

9. 15-16 December 1933, Stanford. Baade and Zwicky give a talk at a meeting of the American Physical Society suggesting the concept of neutron stars, and their origin in supernova explosions.

10. 15 January 1934. The abstract of the talk by Baade and Zwicky is published [30].

\section{References}

[1] Hewish A, Bell S J, Pilkington J D H, Scott P F, Collins R A Nature 217709 (1968)

[2] Haensel P, Potekhin A Y, Yakovlev D G Neutron stars: Equation of State and Structure (New York: Springer, 2007)

[3] Rosenfeld L, in Astrophysics and Gravitation, Proc. Solvay Conference on Physics, 16th (Brussels: Univ. Bruxelles, 1974) p. 174

[4] Baym G, Pethick C J Ann. Rev. Nucl. Sci. 2527 (1975)

[5] Baym G Inst. Phys. Conf. Ser. No. 64, 45 (1982)

[6] Shapiro S L, Teukolsky S A Black Holes, White Dwarfs, and Neutron Stars: The Physics of Compact Objects (New York: Wiley, 1983)

[7] Aksent'eva M S Phys. Usp. 41621 (1998)

[8] Bessarab M Stranitsy Zhizni Landau (Pages of Landau's Life) (Moscow: Moskovskii Rabochii, 1971) [in Russian]

[9] Pais A 1991, Niels Bohr's Times, In Physics, Philosophy, and Polity (Oxford: Clarendon, 1991)

[10] Landau L D Phys. Z. Sowjetunion 1285 (1932)

[11] Hufbauer K Historical Studies in the Physical and Biological Sciences 37337 (2007)

[12] Landau L D, in Collected Papers of L D Landau (Ed. D ter Haar) (London: Pergamon, 1965) p. 60 [reprint of the 1932 paper] 
[13] Landau L D, in Neutron Stars, Black Holes and Binary X-ray Sources (Eds. H Gursky, R Ruffini) (Dordrecht: Reidel, 1974) p. 271 [reprint of 1932 paper]

[14] Nauenberg M J. History of Astronomy 39297 (2008)

[15] Shaviv G The Life of Stars: The Controversial Inception and Emergence of the Theory of Stellar Structure (Berlin: Springer, 2010)

[16] Anderson W Z. Phys. 56851 (1929)

[17] Stoner E C Phil. Mag. 9944 (1930)

[18] Chandrasekhar S Astrophys. J. 7481 (1931)

[19] Bronstein M P Uspekhi Astronomicheskich Nauk 284 (1933) [in Russian]

[20] Landau L, Peierls R Z. Phys. 6956 (1931)

[21] Rutherford E Proc. Roy. Soc. London A 97374 (1920)

[22] Chadwick J Nature 129312 (1932)

[23] Chadwick J, in Proceedings of X International Congress on the History of Science (Paris: Hermann, 1962) vol. 1, p. 159

[24] Curie I, Joliot F Comptes Rendus de l'Académie des Sciences 194273 (1932)

[25] Chadwick J Proc. Roy. Soc. London A 136692 (1932)

[26] Bothe W, Becker H Z. Phys. 66289 (1930)

[27] Curie I Comptes Rendus de l'Académie des Sciences 1931412 (1931)

[28] Webster H C Proc. Roy. Soc. London A 136428 (1932)

[29] Iwanenko D D Nature 129798 (1932)

[30] Baade W, Zwicky F. Phys. Rev. 45138 (1934)

[31] Baade W, Zwicky F Proc. National Acad. Sci. 20254 (1934)

[32] Baade W, Zwicky F Proc. National Acad. Sci. 20259 (1934)

[33] Baade W, Zwicky F Phys. Rev. 4676 (1934)

[34] Kirshner R P Ann. New York Acad. Sci. 30281 (1977)

[35] Rosenfeld L, in Niels Bohr and the Development of Physics (Ed. W Pauli, with the assistance of L Rosenfeld and V Weisskopf) (New York: Pergamon, 1955) p. 70

[36] Milne E A Mon. Not. Roy. Astron. Soc. 92610 (1932)

[37] Chandrasekhar S Mon. Not. Roy. Astron. Soc. 95207 (1935)

[38] Chandrasekhar S Mon. Not. Roy. Astron. Soc. 95227 (1935)

[39] Gamow G Structure of Atomic Nuclei and Nuclear Transformations (Oxford: Oxford University Press, 1937) Chapter XII 
[40] Hund F Ergebn. Exakt. Naturwiss. 15189 (1936)

[41] Landau L D Doklady Akad. Nauk SSSR 17301 (1937) [in Russian]

[42] Landau L D Nature 141333 (1938)

[43] Bohr N, Letter to L Landau on 6 December 1937, in Bohr Letter Collection, Copenhagen

[44] Feinberg E L Priroda (Nature) No. 1, 65 (1998) [in Russian]

[45] Bethe H A Phys. Rev. 55434 (1939)

[46] Thorne K S, Żytkow A N Astrophys. J. 212832 (1977)

[47] Bisnovatyi-Kogan G S, Lamzin S A Sov. Astron. 28187 (1984)

[48] Zimmermann M E, Thorne K S, Eich C, Żytkow A N, Astrophys. J. 346277 (1989)

[49] Barkov M V, Bisnovatyi-Kogan G S, Lamzin S A Astron. Rep. 45230 (2001) 